\title{
Cosmological dark matter in a conformal model
}

\author{
Prasenjit Sanyal, ${ }^{1, *}$ Alekha C. Nayak, ${ }^{2, \dagger}$ Gopal Kashyap, ${ }^{3, \$}$ and Pankaj Jain ${ }^{1, \S}$ \\ ${ }^{1}$ Department of Physics, Indian Institute of Technology, Kanpur 208016, Uttar Pradesh, India \\ ${ }^{2}$ Department of Physics, National Institute of Technology, Shillong, Meghalaya 793003, India \\ ${ }^{3}$ Department of Physics, School of Basic and Applied Sciences, Galgotias University, \\ Sector 17A, Greater Noida 203201, India
}

(Received 17 May 2019; published 18 December 2019)

\begin{abstract}
We study the collider, astrophysical, and cosmological constraints on the dark matter sector of a conformal model within the framework of the freeze-out as well as the freeze-in mechanism. The model has a dark sector with strong self-interactions. This sector couples weakly with the Standard Model particles via a scalar messenger. The lightest dark sector particle is a pionlike fermion antifermion bound state. We find that the model successfully satisfies the constraints coming from the Higgs decay to the visible as well as the invisible sector. We have used the results of the dark matter direct detection experiments, such as XENON1T, in order to impose bounds on the parameters of the model. The model satisfies the indirect detection constraints of gamma ray from the galactic center and Dwarf spheroidal galaxies. We also determine the parameter range for which it satisfies the astrophysical constraints on the dark matter selfcoupling.
\end{abstract}

DOI: $10.1103 /$ PhysRevD.100.115032

\section{INTRODUCTION}

The presence of nonbaryonic dark matter (DM) in the Universe is very well established by cosmological and astrophysical observations. The Planck and Wilkinson Microwave Anisotropy Probe (WMAP) data suggest that dark matter density $\Omega_{X} h^{2}=0.1187 \pm 0.0017 \quad$ [1]. Currently, there are many ground and satellite based experiments looking for direct evidence of dark matter. The direct detection experiments of dark matter are based on elastic scattering of DM with the detector nucleons. No evidence of DM detection is observed till date and there exist rather stringent upper limits on the cross section of dark matter with nucleons from LUX [2,3], PandaX-II [4,5], XENON1T [6], SuperCDMS [7], and CRESST-II [8]. Indirect detection of DM comes through its decay or annihilation at the center of galaxies. Despite several tentative claims of detection, such as, the $1-3 \mathrm{GeV}$ gamma ray excess emission from the galactic center by Fermi-LAT Collaboration [9], so far there does not exist conclusive evidence for a dark matter candidate [10]. Apart from that

\footnotetext{
psanyal@iitk.ac.in

alekhanayak@nitm.ac.in

*gplkumar87@gmail.com

spkjain@iitk.ac.in
}

Published by the American Physical Society under the terms of the Creative Commons Attribution 4.0 International license. Further distribution of this work must maintain attribution to the author(s) and the published article's title, journal citation, and DOI. Funded by SCOAP ${ }^{3}$.
DM may annihilate or decay into monoenergetic $\gamma$-rays. Search for monoenergetic spectral lines in the Fermi-Large Area Telescope (Fermi-LAT) observations produced null results for the DM in the range of $200 \mathrm{MeV}-500 \mathrm{GeV}$. The nonobservation of such a spectral line puts an upper bound (95\% CL) on the annihilation cross section and decay widths of DM candidates [11]. Joint analysis of MAGIC Cherenkov telescopes and Fermi-LAT [12] on gamma ray data from dwarf spheroidal galaxies (dSphs) also imposes an upper bound on DM annihilation cross section in the mass range of $10 \mathrm{GeV}-100 \mathrm{TeV}$.

In this paper, we consider a conformal model discussed earlier [13-17]. This model has self-interacting dark matter sector which is assumed to have a QCD-like dynamics. The action has conformal symmetry and the dark sector communicates with the electroweak sector through a real scalar field $\chi$. The dynamical symmetry breaking of the dark matter sector leads to a vacuum expectation value (VEV) of $\chi$, which in turn induces the electroweak symmetry breaking. In order to handle the strong interaction dynamics, we consider an effective action expressed in terms of dark sector pions $\Pi^{i}$, nucleons, and scalar fields $\Sigma$ and $\chi$. The dark pion acts as the dark matter candidate. The scalar field $\chi$ acts as the messenger field through which the dark pions interact with the Standard Model (SM) sector and $\Sigma$ is the bound state scalar. The three scalar fields, $\Phi, \chi$, and $\Sigma$ mix with one another. Here, $\Phi$ is the Standard Model scalar field which would be equivalent to the Higgs in the absence of mixing with dark sector scalars. After diagonalizing their mass matrix, we obtain three 
physical scalars, $\chi_{1}, \chi_{2}$, and $\chi_{3}$. We identify $\chi_{1}$ as the SM Higgs and $\chi_{2}$ is a massive particle with its mass proportional to the symmetry breaking scale of dark sector. The $\chi_{3}$ particle is classically massless but acquires a mass in quantum theory due to conformal anomaly. We discuss this in more detail below. The dark sector particles $\chi_{2}$ and $\chi_{3}$ decay to the SM sector or to the dark pions within the lifespan of the Universe. For simplicity here, we assume that there exists only a single generation of dark fermions. In this case, we only have one dark pion which acts as a dark matter candidate.

We consider the cosmological implications of this model assuming both a freeze-out and freeze-in scenarios. As we shall see, within the freeze-out scenario, there exists parameter range in which the model satisfies all constraints including the constraint on $\sigma_{\mathrm{DM}} / M_{\mathrm{DM}}$ indicated by some astrophysical observations. Here $\sigma_{\mathrm{DM}}$ is the cross section for DM-DM scattering and $M_{\mathrm{DM}}$ the mass of dark matter particles. We next study the dark matter assuming the freeze-in scenario. We show that this model can accommodate to a very low dark pion mass, as required for a dark matter candidate in this case. In this case, the astrophysical constraint on $\sigma_{\mathrm{DM}} / M$ imposes some limits on the parameters of the model.

The paper is organized as follows: in Sec II, we review the conformal model and identify the different particle states predicted by this model. In Sec. III, we determine the relic density of dark pions in the freeze-out scenario and also impose the collider, direct and indirect DM detection constraints on the conformal model. In Sec. IV, we determine the implications of dark matter assuming the freeze-in scenario. In Sec. V, we show the final allowed space taking all the constraints into account both for freeze-out and freeze-in scenarios. Finally, we conclude in Sec. VI.

\section{REVIEW OF THE CONFORMAL MODEL}

The conformal model introduced in [13-15] has a strongly coupled dark matter sector similar to QCD. The action for the dark sector can be written as

$\mathcal{S}_{D}=\int d^{4} x\left[-\frac{1}{4} G_{\mu \nu}^{a} G^{a \mu \nu}+i \bar{\xi}^{i} \gamma^{\mu} D_{\mu} \xi^{i}-g_{Y}^{\chi} \bar{\xi}^{i} \chi \xi^{i}\right]$,

where $G_{\mu \nu}^{a}$ is the field strength tensor of the dark sector strong interaction mediator, $\xi^{i}$ represent fermion fields, and $\chi$ is a real scalar field. We refer to this strongly coupled sector as hypercolor and call the quarks and gluons in this sector as hyperquarks and hypergluons. In our phenomenological study, we consider only one multiplet of hypercolor fermions but in general there can be several multiplets. The hyperquarks and hypergluons will form bound state dark pions and nucleons. The dark pions may act as dark matter candidates. As we shall see, they are able to satisfy all astrophysical and cosmological constraints.
The dark sector couples to the SM particles by the coupling of $\chi$ to the Higgs sector. The action for the scalar sector of the model can be written as

$$
\begin{aligned}
\mathcal{S}_{S}= & \int d^{4} x\left[\frac{1}{2} g^{\mu \nu} \partial_{\mu} \chi \partial_{\nu} \chi+g^{\mu \nu}\left(D_{\mu} \mathcal{H}\right)^{\dagger}\left(D_{\nu} \mathcal{H}\right)\right. \\
& \left.-\frac{\lambda_{1}}{4}\left(2 \mathcal{H}^{\dagger} \mathcal{H}-\lambda_{2} \chi^{2}\right)^{2}-\frac{\lambda}{4} \chi^{4}\right],
\end{aligned}
$$

where $\mathcal{H}$ is the Higgs multiplet and $D_{\mu}$ is the SM gauge covariant derivative. The Higgs multiplet can be decomposed as

$$
\mathcal{H}=\frac{1}{\sqrt{2}}\left(\begin{array}{l}
\phi_{1}+i \phi_{2} \\
\phi_{3}+i \phi_{4}
\end{array}\right) .
$$

The action has conformal symmetry and we include all terms in the scalar potential which are invariant under this symmetry. In analogy with QCD, the hyperquarks form condensates $\langle\bar{\xi} \xi\rangle$ with effective mass scale $\Lambda_{S}$. Once the condensate is generated, we can substitute it in the dark sector Yukawa terms in the Lagrangian and minimize the potential over the scalar fields $\phi_{3}$ and $\chi$. This generates nonzero values for the VEV of these fields and triggers electroweak symmetry breaking. We denote the VEV of $\phi_{3}$ and $\chi$ as $v_{\mathrm{EW}}$ and $\eta$, respectively. Here $v_{\mathrm{EW}}$ is the electroweak symmetry breaking scale.

The model is interesting since it leads to a selfinteracting dark matter candidate which is preferred by cosmological observations [18-24]. The astrophysical implications of this conformal model have been studied earlier in [25]. Due to scale symmetry, we expect that classically the mass of one of the scalar particles is zero. However, we expect this particle to acquire mass in the full quantum theory due to scale anomaly [26]. We include this scale breaking by adding a term in the effective action [27].

The dark pions and nucleons are potential dark matter candidates. We use an effective model which is similar to the linear sigma model in order to handle these bound state fields. The resulting dark sector effective Lagrangian can be written as

$$
\begin{aligned}
\mathcal{L}_{\Sigma}= & \bar{\Psi} i \gamma^{\mu} \partial_{\mu} \Psi+\frac{1}{2} \partial^{\mu} \Sigma \partial_{\mu} \Sigma+\frac{1}{2} \partial^{\mu} \Pi \partial_{\mu} \Pi \\
& -g_{\Psi} \bar{\Psi}\left(\Sigma+i \Pi \gamma_{5}\right) \Psi-\frac{\lambda_{5}}{4}\left(\Sigma^{2}+\Pi^{2}-\lambda_{6} \chi^{2}\right)^{2},
\end{aligned}
$$

where $\Pi, \Psi$, and $\Sigma$ denote the dark sector pions, nucleons, and the scalar fields, respectively. This model becomes same as the linear sigma model if we replace the $\chi$ field with its vacuum expectation value. Here we have chosen this Lagrangian because it satisfies conformal invariance. So far the model has chiral symmetry, which is not a symmetry of the hypercolor interactions, due to the presence of the Yukawa terms. We break this symmetry by adding the following term: 


$$
\mathcal{L}_{\Pi}=-\frac{\lambda_{7}}{2} \Pi^{2} \chi^{2}
$$

With the addition of this term, the dark sector pions acquire mass.

We next need to minimize the scalar potential given in Eqs. (2) and (4). So far, we have maintained scale invariance in the effective action. However, even though the original action Eq. (1) is classically scale invariant, the scale invariance is broken due to the anomaly [26]. Hence, the effective action which represents the low energy dynamics of the bound states need not have this symmetry. We also need to break this symmetry in order to obtain a nontrivial minimum of the potential without fine-tuning any parameter to zero [16]. We break the scale symmetry by modifying the potential term for $\chi$ such that [27]

$$
\frac{\lambda}{4} \chi^{4} \rightarrow \frac{\lambda}{4} \chi^{4} \log \left(\frac{\chi^{2}}{\Lambda^{2}}\right)
$$

where $\Lambda$ is a dimensional parameter related to the strong interaction scale $\Lambda_{S}$. With this modification, the potential acquires a nontrivial minimum with nonzero vacuum expectation values for the fields $\Sigma$ and $\chi$. The scale breaking terms lead to a mass term for the dilaton which would be massless in the limit of exact scale invariance.

The minimum of the dark sector potential occurs at

$$
\Sigma^{2}+\Pi^{2}=\lambda_{6} \eta^{2}
$$

with

$$
\begin{aligned}
& \langle\chi\rangle=\eta=\Lambda / \exp (1 / 4), \quad\left\langle\phi_{3}\right\rangle=v_{\mathrm{EW}}, \\
& \langle\Sigma\rangle=v_{D}, \quad\langle\Pi\rangle=0,
\end{aligned}
$$

where $v_{D}=\sqrt{\lambda_{6}} \eta$. We expand the fields $\phi_{3}, \chi, \Sigma$, and $\Pi$ around their VEV,

$\phi_{3}=v_{\mathrm{EW}}+\hat{\phi}, \quad \chi=\eta+\hat{\chi}, \quad \Sigma=v_{D}+\sigma, \quad \Pi=\pi$.

From the scalar potential (2), and dark sector potential (4), we obtain mixing between the fields $\hat{\phi}, \sigma$, and $\hat{\chi}$, having the following squared mass matrix:

$\mathcal{M}^{2}=\left(\begin{array}{ccc}2 \lambda_{1} \lambda_{2} & 0 & -2 \lambda_{1} \lambda_{2}^{3 / 2} \\ 0 & 2 \lambda_{5} \lambda_{6} & -2 \lambda_{5} \lambda_{6}^{3 / 2} \\ -2 \lambda_{1} \lambda_{2}^{3 / 2} & -2 \lambda_{5} \lambda_{6}^{3 / 2} & 2\left(\lambda_{1} \lambda_{2}^{2}+\lambda_{5} \lambda_{6}^{2}\right)+\frac{m^{2}}{\eta^{2}}\end{array}\right) \eta^{2}$,

where $m^{2}=2 \lambda \eta^{2}$ is the mass term generated by the contributions due to scale anomaly. The eigenvalues of this mass matrix gives us three physical scalar particles, which we denote as $\chi_{1}, \chi_{2}$, and $\chi_{3}$. We identify $\chi_{1}$ as the
$125 \mathrm{GeV}$ Higgs Boson. The mass matrix can be diagonalized by an orthogonal matrix $R$ with three Euler angles $\left(\alpha_{1}, \alpha_{2}, \alpha_{3}\right)$,

$$
\begin{aligned}
& R\left(\alpha_{1}, \alpha_{2}, \alpha_{3}\right) \\
& \quad=\left(\begin{array}{ccc}
c_{\alpha_{1}} c_{\alpha_{2}} & -s_{\alpha_{1}} c_{\alpha_{2}} & s_{\alpha_{2}} \\
-c_{\alpha_{1}} s_{\alpha_{2}} s_{\alpha_{3}}+s_{\alpha_{1}} c_{\alpha_{3}} & c_{\alpha_{1}} c_{\alpha_{3}}+s_{\alpha_{1}} s_{\alpha_{2}} s_{\alpha_{3}} & c_{\alpha_{2}} s_{\alpha_{3}} \\
-c_{\alpha_{1}} s_{\alpha_{2}} c_{\alpha_{3}}-s_{\alpha_{1}} s_{\alpha_{3}} & -c_{\alpha_{1}} s_{\alpha_{3}}+s_{\alpha_{1}} s_{\alpha_{2}} c_{\alpha_{3}} & c_{\alpha_{2}} c_{\alpha_{3}}
\end{array}\right)
\end{aligned}
$$

and the mass eigenstates are obtained such that

$$
\left(\begin{array}{l}
\phi \\
\sigma \\
\chi
\end{array}\right)=R\left(\begin{array}{l}
\chi_{1} \\
\chi_{2} \\
\chi_{3}
\end{array}\right) .
$$

The parameters of the model $\left(\lambda_{1}, \lambda_{2}, \lambda_{5}, \lambda_{6}, \lambda_{7}, m^{2}\right)$, written in terms of the physical masses, Euler angles (or the mixing angles), and the VEVs are

$$
\begin{aligned}
\lambda_{1}= & \frac{\left(M_{\chi_{1}}^{2} R_{11}^{2}+M_{\chi_{2}}^{2} R_{12}^{2}+M_{\chi_{3}}^{2} R_{13}^{2}\right)^{3}}{2 \eta^{2}\left(M_{\chi_{1}}^{2} R_{11} R_{31}+M_{\chi_{2}}^{2} R_{12} R_{32}+M_{\chi_{3}}^{2} R_{13} R_{33}\right)^{2}} \\
\lambda_{2}= & \frac{\left(M_{\chi_{1}}^{2} R_{11} R_{31}+M_{\chi_{2}}^{2} R_{12} R_{32}+M_{\chi_{3}}^{2} R_{13} R_{33}\right)^{2}}{\left(M_{\chi_{1}}^{2} R_{11}^{2}+M_{\chi_{2}}^{2} R_{12}^{2}+M_{\chi_{3}}^{2} R_{13}^{2}\right)^{2}} \\
\lambda_{5}= & \frac{\left(M_{\chi_{1}}^{2} R_{21}^{2}+M_{\chi_{2}}^{2} R_{22}^{2}+M_{\chi_{3}}^{2} R_{23}^{2}\right)^{3}}{2 \eta^{2}\left(M_{\chi_{1}}^{2} R_{21} R_{31}+M_{\chi_{2}}^{2} R_{22} R_{32}+M_{\chi_{3}}^{2} R_{23} R_{33}\right)^{2}} \\
\lambda_{6}= & \frac{\left(M_{\chi_{1}}^{2} R_{21} R_{31}+M_{\chi_{2}}^{2} R_{22} R_{32}+M_{\chi_{3}}^{2} R_{23} R_{33}\right)^{2}}{\left(M_{\chi_{1}}^{2} R_{21}^{2}+M_{\chi_{2}}^{2} R_{22}^{2}+M_{\chi_{3}}^{2} R_{23}^{2}\right)^{2}} \\
\lambda_{7}= & \frac{M_{\pi}^{2}}{\eta^{2}} \\
m^{2}= & M_{\chi_{1}}^{2} R_{31}^{2}+M_{\chi_{2}}^{2} R_{32}^{2}+M_{\chi_{3}}^{2} R_{33}^{2} \\
& -\left(M_{\chi_{1}}^{2} R_{11}^{2}+M_{\chi_{2}}^{2} R_{12}^{2}+M_{\chi_{3}}^{2} R_{13}^{2}\right) \lambda_{2} \\
& -\left(M_{\chi_{1}}^{2} R_{21}^{2}+M_{\chi_{2}}^{2} R_{22}^{2}+M_{\chi_{3}}^{2} R_{23}^{2}\right) \lambda_{6} .
\end{aligned}
$$

The VEVs are related by the equations

$$
v_{D}^{2}=\lambda_{6} \eta^{2} \quad \text { and } \quad v_{\mathrm{EW}}^{2}=\lambda_{2} \eta^{2} .
$$

The mass of the physical scalars $\left(\chi_{1}, \chi_{2}, \chi_{3}\right)$ are related by the relation

$$
M_{\chi_{1}}^{2} R_{11} R_{21}+M_{\chi_{2}}^{2} R_{12} R_{22}+M_{\chi_{3}}^{2} R_{13} R_{23}=0 .
$$

The independent parameters of the model are $\alpha_{1}, \alpha_{2}, \alpha_{3}$, $M_{\chi_{1}}, M_{\chi_{2}}, M_{\pi}$, and $v_{\mathrm{EW}}$, and we set $M_{\chi_{1}}$ and $v_{\mathrm{EW}}$ to be 125 and $246 \mathrm{GeV}$, respectively. We take $\chi_{1}$ to be the lightest observed Higgs and assume $\chi_{2}$ and $\chi_{3}$ heavier than $\chi_{1}$. Due to scale anomaly, $\chi_{3}$ acquires a mass which we assume to be larger than the mass of the Higgs boson. The Feynman rules 
and relevant parameters in the model are implemented with FeynRules [28].

\section{PHENOMENOLOGY: FREEZE-OUT SCENARIO}

In this section, we discuss the phenomenological implications of our model assuming that the relic density of DM is obtained in the freeze-out scenario. We investigate the constraints from the Higgs sector such as the branching fraction of the Higgs to the visible sector and the upper bound on the Higgs decay to the invisible sector. We also explore the spin-independent (SI) scattering cross section of pions with the nucleons and the $\gamma$-ray constraints termed as direct and indirect detection of DM, respectively. For simplicity, we considered the mixing angles in the region $\left[0, \frac{\pi}{2}\right]$. For the dark pion mass, we consider $M_{\pi} \in$ [50-400] GeV and, as we shall see, some of the allowed parameter space lies close to the resonance of $\chi_{1}, \chi_{2}$, and $\chi_{3}$. From the perspective of phenomenology, the dark sector neutral scalars $\chi_{2}$ and $\chi_{3}$ share the same Higgs-like properties with mass greater than $125 \mathrm{GeV}$. Due to the presence of additional two Higgs-like scalars, the analysis of our model has some similarities with Higgs portal DM [29-33].

\section{A. Collider constraints}

The deviations from the SM can be implemented by introducing the coupling modifiers $\left(\kappa^{\prime} s\right)$,

$$
g_{\chi_{1} f \bar{f}}=\kappa_{f} g_{h f \bar{f}}^{\mathrm{SM}} \quad \text { and } \quad g_{\chi_{1} V V}=\kappa_{V} g_{h V V}^{\mathrm{SM}},
$$

such that $\kappa_{f}=1$ and $\kappa_{V}=1$ in SM. Here $g_{h f \tilde{f}}^{\mathrm{SM}}$ and $g_{h V V}^{\mathrm{SM}}$ represent the SM Higgs couplings to fermions and vector

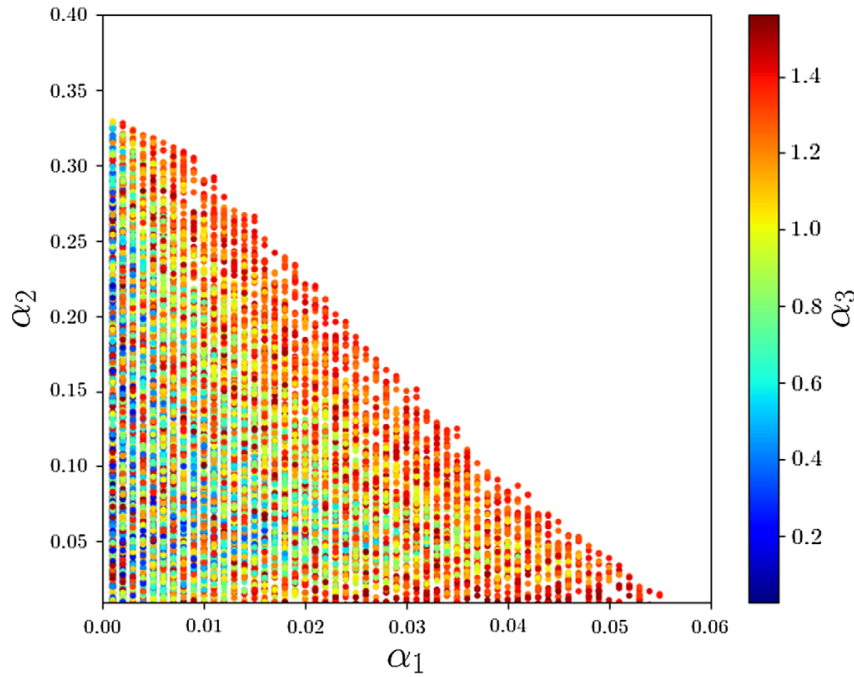

(a) bosons, respectively, and $g_{\chi_{1} f \bar{f}}$ and $g_{\chi_{1} V V}$ represent the corresponding couplings in our model. The coupling modifiers are given by $\kappa_{f}=\kappa_{V}=\cos \alpha_{1} \cos \alpha_{2}$. The constraints on these parameters from the Higgs coupling measurements are given in $[34,35]$. Using the latest beta version of Higgs signals [36], we have imposed the constraints on the mixing angles as shown in Figs. 1(a) and 1(b). For $M_{\pi} \geq M_{\chi_{1}} / 2$, the allowed region in the $\alpha_{1}-\alpha_{2}$ plane is a radius of approximately 0.32 radians, leaving $\alpha_{3}$ unconstrained. The invisible decay of Higgs opens up when $M_{\pi}$ goes below $M_{\chi_{1}} / 2$; we can express this branching fraction as

$$
\mathrm{Br}^{\mathrm{inv}}=\frac{\Gamma^{\chi_{1} \rightarrow \pi \pi}}{\Gamma^{\chi_{1} \rightarrow \pi \pi}+R_{11}^{2} \Gamma^{\mathrm{SM}}},
$$

where $\Gamma^{\mathrm{SM}}=4.07 \mathrm{MeV}$ is the SM Higgs decay width. The observed upper limit on Higgs invisible branching fraction is $0.24[37,38]$. The decay width of $\chi_{1}$ to dark pions is given by

$$
\Gamma \chi_{1} \rightarrow \pi \pi=\frac{g_{\chi_{1} \pi \pi}^{2}}{32 M_{\chi_{1}} \pi} \sqrt{1-\frac{4 M_{\pi}^{2}}{M_{\chi_{1}}^{2}}} .
$$

We see that the mixing angles are much constrained when the Higgs invisible decay to dark pions is open.

\section{B. Relic density calculation}

In this section, we calculate the relic density of dark pions within the framework of freeze-out scenario. As explained earlier, the dark sector of our model contains dark pions and scalar particles $\left(\chi_{2}\right.$ and $\left.\chi_{3}\right)$. The scalar particles have masses of the order of the dark strong interaction scale. These decay into dark pions early in the Universe, leaving pions as the only DM candidate. The total decay

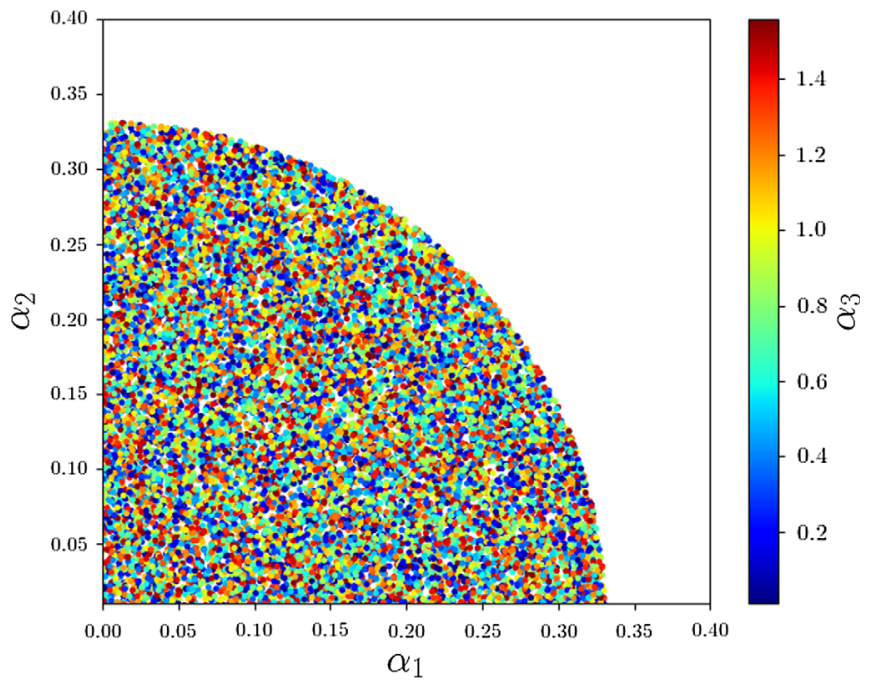

(b)

FIG. 1. The $1 \sigma$ allowed region of mixing angles is shown. (a) shows the allowed region for $M_{\pi}=60 \mathrm{GeV}$ and $M_{\chi_{2}}=500 \mathrm{GeV}$. (b) shows the allowed region for $M_{\pi} \geq M_{\chi_{1}} / 2$. 


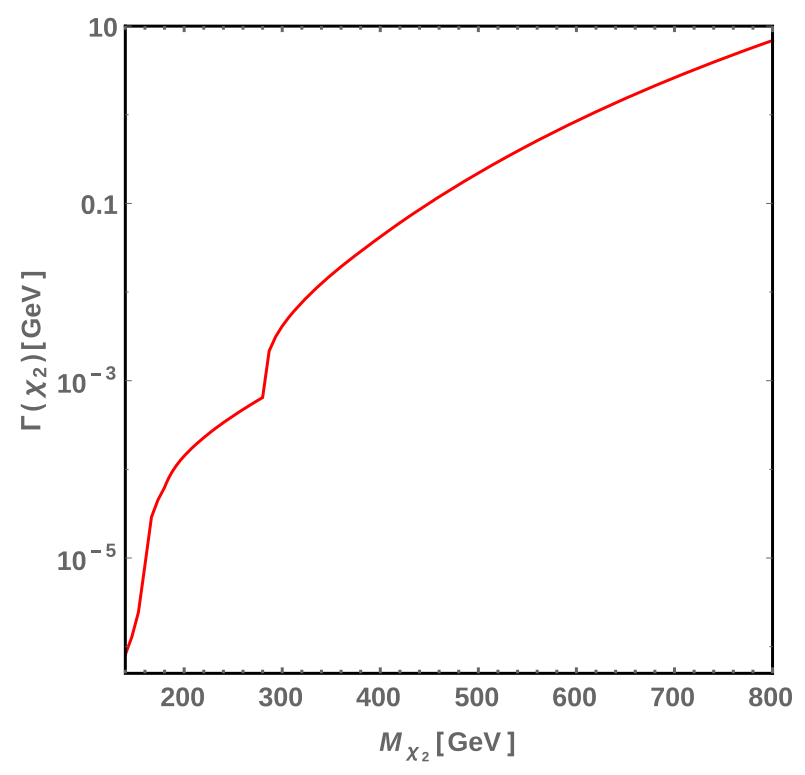

(a)

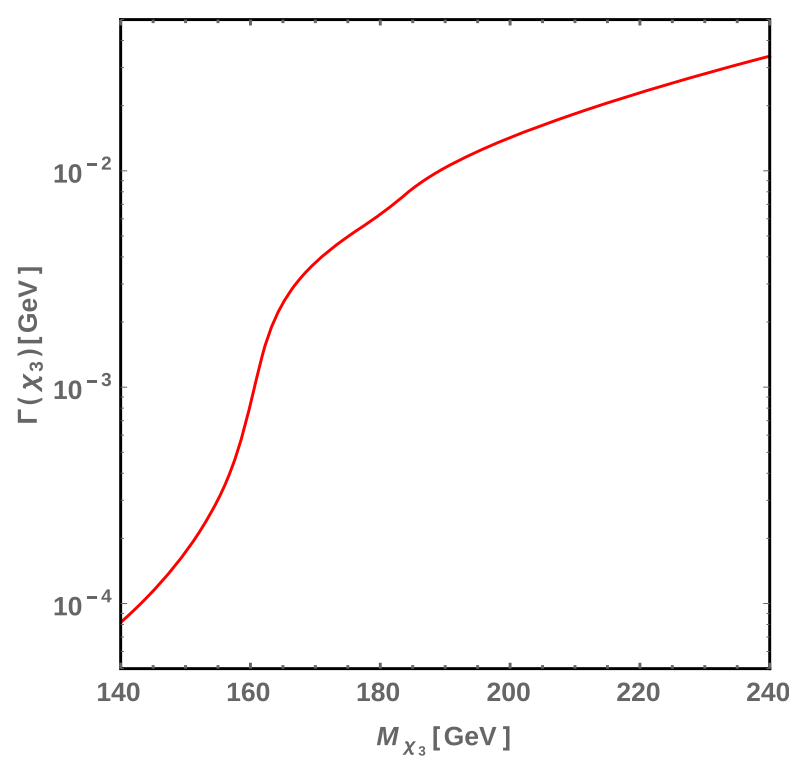

(b)

FIG. 2. The decay width of $\chi_{2}$ and $\chi_{3}$ for $\alpha_{1}=0.01$ radians, $\alpha_{2}=0.1$ radians, $\alpha_{3}=1.0$ radians, and dark pion mass of $200 \mathrm{GeV}$. The decay width is sufficiently large compared to $H_{0}$.

width of $\chi_{2}$ and $\chi_{3}$ as a function of their masses is computed using HDECAY [39] and CalcHEP [40] and plotted in Fig. 2. We require that the lifetime of $\chi_{2}$ and $\chi_{3}$ be smaller than the lifetime of the Universe $\left(H_{0}^{-1}\right)$, i.e., $\Gamma_{\chi_{2}}$ and $\Gamma_{\chi_{3}}$ be greater than $H_{0}$, which is satisfied in this case, as shown in Fig. 2. We solve the Boltzmann equation to find the freezeout temperature and the comoving number density of dark pions. The Boltzmann equation [41,42] for comoving number density of dark pions can be written as

$$
\frac{d Y}{d x}=-\left(\frac{45 G}{\pi}\right)^{-1 / 2} \frac{g_{\star}^{1 / 2} M_{\pi}}{x^{2}}\langle\sigma v\rangle\left(Y^{2}-Y_{\mathrm{eq}}^{2}\right),
$$

where $Y=\frac{n_{\pi}}{s}, x=\frac{M_{\pi}}{T}, s$ is the entropy density, and $g_{\star}$ is the number of degrees of freedom. This is given by

$$
g_{\star}^{1 / 2}=\frac{h_{s}(T)}{g_{\rho}(T)^{1 / 2}}\left(1+\frac{1}{3} \frac{T}{h_{s}(T)} \frac{d h_{s}(T)}{d T}\right),
$$

where $g_{s}(T)$ and $g_{\rho}(T)$ are the effective degrees of freedom related to entropy and energy density, respectively. Thermal average cross section $\langle\sigma v\rangle$ is given by

$$
\langle\sigma v\rangle=\frac{1}{8 M_{\pi}^{4} T K_{2}^{2}(x)} \int_{4 M_{\pi}^{2}}^{\infty} d s \sigma\left(s-4 M_{\pi}^{2}\right) \sqrt{s} K_{1}\left(\frac{\sqrt{s}}{T}\right),
$$

where $K_{1}$ is the Bessel function. Integrating Eq. (19) from $x=0$ to $\frac{M_{\pi}}{T_{0}}$, where $T_{0}=2.72 \mathrm{~K}$ is the temperature of the cosmic microwave background radiation, we get the current value of $Y$, i.e., $Y_{0}$, and hence the relic density. The present relic density is given by

$$
\Omega_{\pi} h^{2}=2.755 \times 10^{8}\left(\frac{M_{\pi}}{\mathrm{GeV}}\right) Y_{0}
$$

We use the MicrOMEGAs package [43] to calculate the relic density of dark pion. Here $\pi \pi \rightarrow \chi_{1,2,3} \rightarrow$ SM SM processes contribute to the total thermally averaged cross section. The value of this cross section at the decoupling temperature determines the relic density of the dark pion and the s-channel processes involved in the cross section might encounter poles. We find that the process $\pi \pi \rightarrow$ $\chi_{1,2,3} \rightarrow$ SM SM also gets resonant contributions when the mass $M_{\pi}$ is close to half the mass of the scalars $\left(\chi_{1}, \chi_{2}\right.$, and $\left.\chi_{3}\right)$. As a result of the resonance, there is a peak in $\langle\sigma v\rangle$ characterized by the parameter $\epsilon=\Gamma_{\text {res }} / M_{\text {res }}$, where $M_{\text {res }}$ is the mass of the resonance particles $\chi_{1}, \chi_{2}$, and $\chi_{3}$. This leads to an enhancement in comparison to the nonresonant cross section. ${ }^{1}$ Enhancement of annihilation cross section at resonance has been discussed in great details in Refs. [44-47]. The enhancement of the cross section at resonance leads to a sudden drop of relic density.

\section{Direct detection and indirect detection constraints}

Direct detection experiments impose strong limits on the interaction of dark matter particles with nucleons. In these experiments, dark matter particles are scattered elastically

\footnotetext{
${ }^{1}$ The most common approach of Taylor expansion of $\langle\sigma v\rangle=$ $a+b v^{2}$ and then the substitution of $v^{2}=6 / x$ does not hold at resonance as shown in Ref. [44]. The numerical result of $\langle\sigma v\rangle$ differs sharply from the approximate result obtained from Taylor expansion and the disagreement is sharper for smaller $\epsilon$.
} 


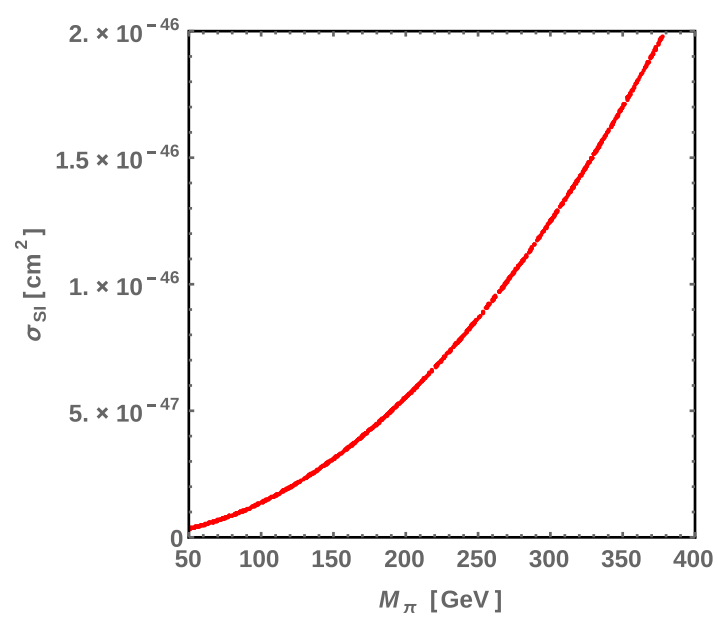

(a)

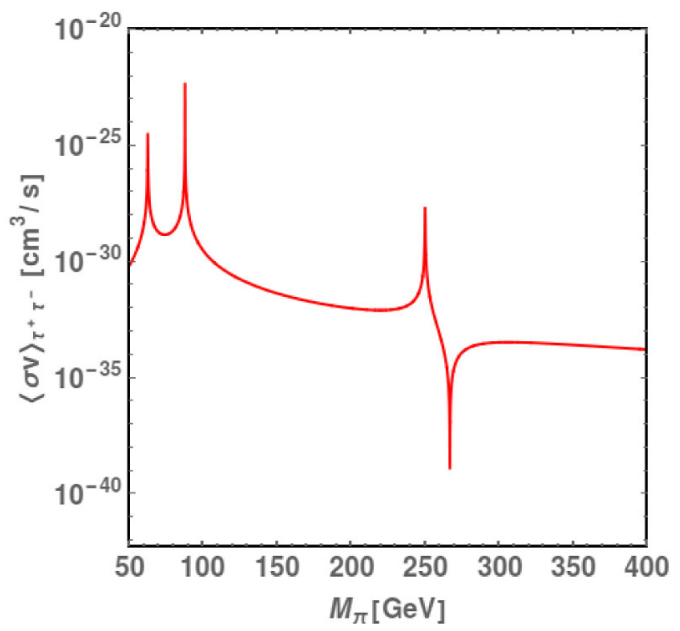

(c)

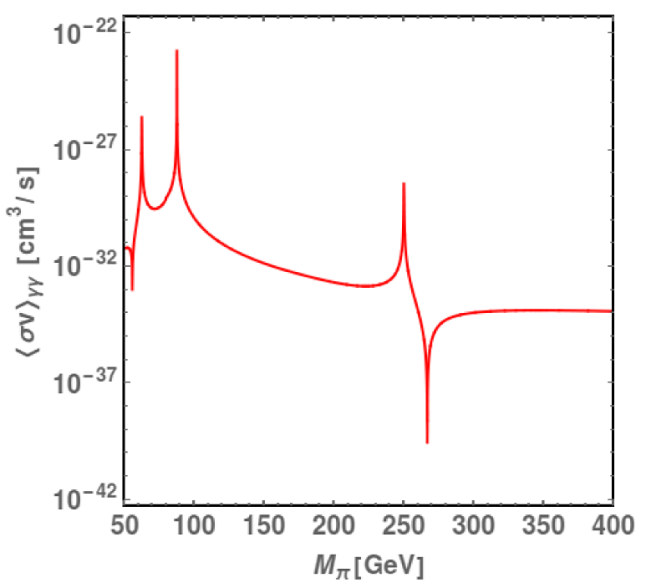

(e)

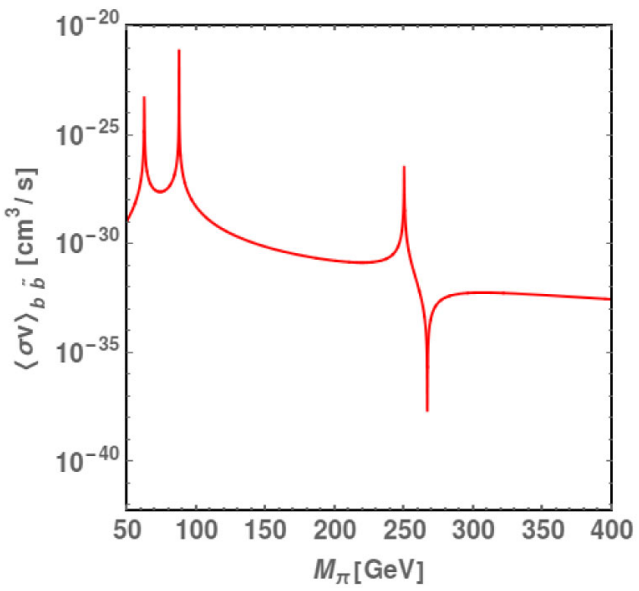

(b)

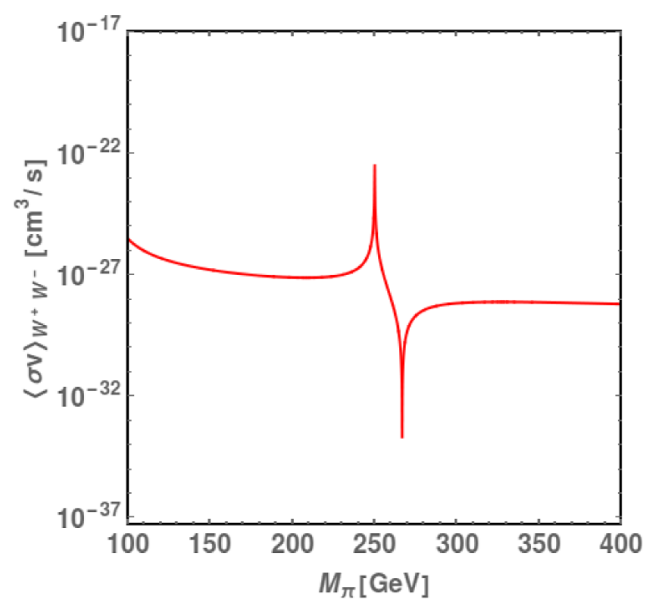

(d)

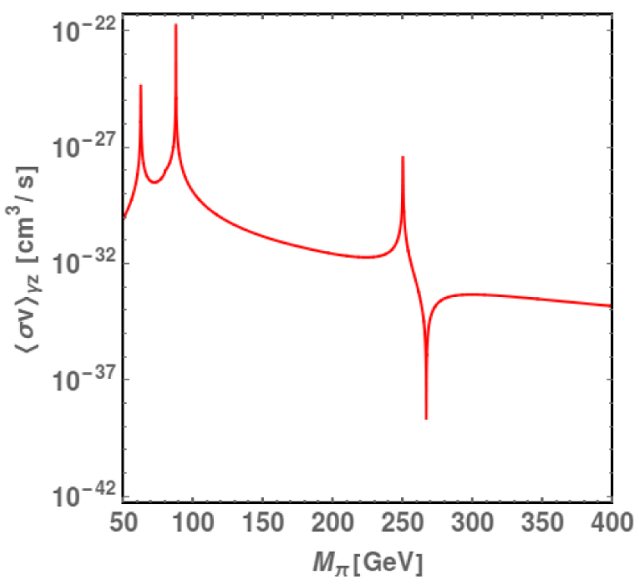

(f)

FIG. 3. (a) The dark pion-nucleon scattering cross section is shown for dark pion mass in the range [50-400] GeV for the mixing angles $\alpha_{1}=0.01$ radians, $\alpha_{2}=0.1$ radians, $\alpha_{3}=1.0$ radians, and $M_{\chi_{2}}=500 \mathrm{GeV}$. For the same set of parameters, (b)-(f) show the cross sections for dark pion annihilation into $b \bar{b}, \tau^{+} \tau^{-}, W^{+} W^{-}, \gamma \gamma$, and $\gamma Z$, respectively. We see from these plots that the $\gamma$-ray constraints from dSphs and galactic center are automatically satisfied and are much weaker than the direct detection constraints. The peaks appear due to the Breit-Wigner resonance of $\chi_{1}, \chi_{2}, \chi_{3}$. The troughs in these plots arise since some coupling factors cross zero at the corresponding values of the pion mass. 
with the nucleons present in the detectors and the recoil energy is detected. Experiments can be sensitive to both nuclear SI interactions and spin-dependent interactions, but current experiments are more sensitive to SI interactions. The results obtained from LUX [2,3] and PandaX-II [4,5] put upper bounds on SI scattering cross section. Latest result obtained from XENON1T [6] experiment being more restrictive excludes a large parameter space in many DM models as the upper limit on the SI cross sections gets pushed to an order of $10^{-47} \mathrm{~cm}^{2}$ for DM mass of around $50 \mathrm{GeV}$ at $90 \%$ confidence limit. These results impose a very strong constraint, particularly close to the Higgs resonance $\left(M_{\pi} \sim 60 \mathrm{GeV}\right)$.

The expression for SI elastic scattering cross section between dark pion and nucleon $(\mathrm{N})$ through the exchange of $\chi_{1}, \chi_{2}$, and $\chi_{3}$ is given by

$$
\sigma_{\mathrm{SI}}^{\pi-N}=\frac{\mu_{\pi N}^{2} m_{N}^{2} f^{2}}{\pi M_{\pi}^{2} v_{\mathrm{EW}}^{2}}\left(\frac{R_{11} g_{\chi_{1} \pi \pi}}{M_{\chi_{1}^{2}}}+\frac{R_{12} g_{\chi_{2} \pi \pi}}{M_{\chi_{2}^{2}}}+\frac{R_{13} g_{\chi_{3} \pi \pi}}{M_{\chi_{3}^{2}}}\right)^{2},
$$

where $f \sim 0.3[48]$ is the usual nucleonic matrix element and $\mu_{\pi N}$ is the reduced mass of dark pion and nucleon. We compute the DM-nucleon SI cross section using MicrOMEGAs. The SI cross section of dark pions with the nucleons is plotted in Fig. 3 for pion $\in[50,400] \mathrm{GeV}$.

We next turn to the indirect detection of DM where we primarily look into the gamma ray signals from the dSphs and monoenergetic spectrum from the galactic center. The diffused gamma ray search studied by the combined analysis of Fermi-LAT and MAGIC observations puts limits on the DM scattering cross section to $b \bar{b}, W^{+} W^{-}$, $\tau^{+} \tau^{-}$, and $\mu^{+} \mu^{-}$. The cross sections are given by

$$
\langle\sigma v\rangle_{f \bar{f}}=\frac{N_{c}}{4 \pi}\left(\frac{m_{f}}{v_{\mathrm{EW}}}\right)^{2}\left|\frac{R_{11} g_{\chi_{1} \pi \pi}}{s-M_{\chi_{1}}^{2}+i M_{\chi_{1}} \Gamma_{\chi_{1}}}+\frac{R_{12} g_{\chi_{2} \pi \pi}}{s-M_{\chi_{2}}^{2}+i M_{\chi_{2}} \Gamma_{\chi_{2}}}+\frac{R_{13} g_{\chi_{3} \pi \pi}}{s-M_{\chi_{3}}^{2}+i M_{\chi_{3}} \Gamma_{\chi_{3}}}\right|^{2}\left(1-\frac{4 m_{f}^{2}}{s}\right)^{3 / 2},
$$

where $N_{c}$ is the color charge of the fermion $f$.

$$
\begin{aligned}
\langle\sigma v\rangle_{W^{+} W^{-}}= & \frac{s}{2 \pi v_{\mathrm{EW}}^{2}}\left|\frac{R_{11} g_{\chi_{1} \pi \pi}}{s-M_{\chi_{1}}^{2}+i M_{\chi_{1}} \Gamma_{\chi_{1}}}+\frac{R_{12} g_{\chi_{2} \pi \pi}}{s-M_{\chi_{2}}^{2}+i M_{\chi_{2}} \Gamma_{\chi_{2}}}+\frac{R_{13} g_{\chi_{3} \pi \pi}}{s-M_{\chi_{3}}^{2}+i M_{\chi_{3}} \Gamma_{\chi_{3}}}\right|^{2} \\
& \times \sqrt{1-\frac{4 M_{W}^{2}}{s}}\left(1-\frac{4 M_{W}^{2}}{s}+\frac{3}{4}\left(\frac{4 M_{W}^{2}}{s}\right)^{2}\right) .
\end{aligned}
$$

The dark pion annihilation to $\gamma \gamma$ and $Z \gamma$ is important for detection of monochromatic gamma ray signal from the galactic center. The cross sections for these processes are loop suppressed and are given by [49]

$$
\langle\sigma v\rangle_{\gamma \gamma / \gamma Z}=\frac{8}{\sqrt{s}}\left|\frac{R_{11} g_{\chi_{1} \pi \pi}}{\left(s-M_{\chi_{1}}^{2}\right)+i M_{\chi_{1}} \Gamma_{\chi_{1}}}+\frac{R_{12} g_{\chi_{2} \pi \pi}}{\left(s-M_{\chi_{2}}^{2}\right)+i M_{\chi_{2}} \Gamma_{\chi_{2}}}+\frac{R_{13} g_{\chi_{3} \pi \pi}}{\left(s-M_{\chi_{3}}^{2}\right)+i M_{\chi_{3}} \Gamma_{\chi_{3}}}\right|^{2} \Gamma_{\gamma \gamma / \gamma Z}(s),
$$

where $\Gamma_{\gamma \gamma / \gamma Z}(s)$ is computed by replacing the scalar mass square in the Higgs decay rate into $\gamma \gamma / \gamma Z$ with $s$ [50-52]. In the nonrelativistic limit, we can consider the pions to be almost at rest (which is a very good approximation) and in this limit $s \sim 4 M_{\pi}^{2}$. The results are consistent with MicrOMEGAs and cross sections are shown in Fig. 3. The DM distribution in the dSphs are parametrized following a Navarro-Frenk-White (NFW) profile [53], whereas for the galactic DM halo we consider the Einasto [54] profile as it is more restrictive compared to the NFW profile.

\section{Dark matter self-interaction constraints}

The observed central densities of dark matter halos of a wide range of the astrophysical objects from dwarf galaxies to galaxy clusters have lesser density compared to the prediction from the collisionless cold dark matter N-body simulations [55]. This mass deficit anomaly could be resolved if the cold dark matter particles undergo elastic scattering among one another. The self-interacting dark matter leads to exchange of heat energy between inner and outer halos and leads to a lower density of the inner halos [56-59]. Hence, the self-interaction of dark matter is able solve this too-big-to-fail problem $[60,61]$ and also the cusp vs core problem [55,62-64]. Astrophysical observations suggest the following value for DM-DM scattering cross section $\left(\sigma_{\mathrm{DM}}\right)[22]$ :

$$
\frac{\sigma_{\mathrm{DM}}}{M_{\mathrm{DM}}} \sim 1.5 \mathrm{~cm}^{2} \mathrm{~g}^{-1},
$$

where $M_{\mathrm{DM}}$ is the mass of the dark matter. This estimate is subject to some uncertainties. There also exists an upper bound $[21,22,56,57,65,66]$,

$$
\frac{\sigma_{\mathrm{DM}}}{M_{\mathrm{DM}}} \lesssim 1 \mathrm{~cm}^{2} \mathrm{~g}^{-1},
$$


obtained from different astrophysical observations. These considerations suggest that the dark pion-dark pion $(\pi \pi \rightarrow \pi \pi)$ scattering cross section $\sigma_{\pi}$ should satisfy $\sigma_{\mathrm{DM}} / M_{\mathrm{DM}} \in[4.7-7.0] \times 10^{3} \mathrm{GeV}^{-3}[67]$.

\section{PHENOMENOLOGY: FREEZE-IN SCENARIO}

In this section, we examine the implications of the model assuming the freeze-in scenario [68-70]. In this scenario, DM particles are never in equilibrium with the cosmic plasma and at very high temperature their density is zero. The production of dark pions happens through SM SM $\rightarrow$ $\pi \pi$ and $\chi_{1} \rightarrow \pi \pi$ processes. The freeze-in scenario within the Higgs portal DM models successfully explains the astrophysical constraints coming from DM self-interaction $[67,71,72]$. As we shall see, this is also true in our conformal model. We will use self-interacting DM scattering constraints to put limits on the parameter space.

In the case of freeze-in, we find that we can satisfy all the constraints provided we choose both the parameters $\lambda_{2}$ and $\lambda_{6}$ relatively small. We shall assume $\lambda_{2} \ll \lambda_{6} \ll 1$ which leads to $\eta \gg v_{D} \gg v_{\mathrm{EW}}$. We also need to choose $\lambda_{7} \ll 1$ due to the low value of pion mass in this scenario. The low value of $\lambda_{7}$ does not require any fine-tuning since this parameter corresponds to a symmetry breaking term. Furthermore, the small values of $\lambda_{2}$ and $\lambda_{6}$ also do not require fine-tuning since these do not acquire large contributions at loop orders. In the limit $\lambda_{2} \ll \lambda_{6} \ll 1$, we can diagonalize the mass matrix, Eq. (10), perturbatively and relate the particles $\hat{\phi}, \sigma$, and $\hat{\chi}$ to the physical particles $\chi_{1}$, $\chi_{2}$, and $\chi_{3}$. At leading order, we obtain

$$
\begin{aligned}
& \hat{\phi} \approx \frac{1}{\sqrt{1+a_{3}^{2}}} \chi_{1}+\frac{c_{1}}{\sqrt{1+c_{1}^{2}+c_{2}^{2}}} \chi_{3} \\
& \sigma \approx \frac{1}{\sqrt{1+b_{3}^{2}}} \chi_{2}+\frac{c_{2}}{\sqrt{1+c_{1}^{2}+c_{2}^{2}}} \chi_{3} \\
& \hat{\chi} \approx \frac{1}{\sqrt{1+c_{1}^{2}+c_{2}^{2}}} \chi_{3}+\frac{a_{3}}{\sqrt{1+a_{3}^{2}}} \chi_{1}+\frac{b_{3}}{\sqrt{1+b_{3}^{2}}} \chi_{2},
\end{aligned}
$$

where $c_{1}, c_{2}, a_{3}$, and $b_{3}$ are all small compared to unity and given by

$$
\begin{aligned}
& c_{1}=\frac{M_{\phi \chi}}{M_{\chi}^{2}-M_{\phi}^{2}}, \\
& c_{2}=\frac{M_{\sigma \chi}}{M_{\chi}^{2}-M_{\sigma}^{2}}, \\
& a_{3}=\frac{M_{\phi \chi}}{M_{\phi}^{2}-M_{\chi}^{2}}, \\
& b_{3}=\frac{M_{\sigma \chi}}{M_{\sigma}^{2}-M_{\chi}^{2}}
\end{aligned}
$$

and

$$
\begin{aligned}
M_{\phi}^{2} & =2 \lambda_{1} \lambda_{2} \eta^{2} \approx M_{\chi_{1}}^{2}, \\
M_{\sigma}^{2} & =2 \lambda_{5} \lambda_{6} \eta^{2} \approx M_{\chi_{2}}^{2}, \\
M_{\chi}^{2} & \approx m^{2} \approx M_{\chi_{3}}^{2}, \\
M_{\phi \chi} & =-2 \lambda_{1} \lambda_{2}^{3 / 2} \eta^{2}, \\
M_{\sigma \chi} & =-2 \lambda_{5} \lambda_{6}^{3 / 2} \eta^{2} .
\end{aligned}
$$

Here we have assumed that $2\left(\lambda_{1} \lambda_{2}^{2}+\lambda_{5} \lambda_{6}^{2}\right) \ll m^{2} / \eta^{2}$. The masses of the three physical scalars $\chi_{1}, \chi_{2}$, and $\chi_{3}$ are $M_{\chi_{1}}$, $M_{\chi_{2}}$, and $M_{\chi_{3}}$, respectively, and $M_{\pi}=\sqrt{\lambda_{7}} \eta$. It is clear that $M_{\chi_{2}} \gg M_{\chi_{1}}$. Furthermore, we shall choose $m$ such that $M_{\chi_{3}} \gg M_{\chi_{2}}$.

In the freeze-in scenario, the relic density of dark matter, i.e., dark pions, is given by $[71,73]$

$$
\frac{\Omega_{\mathrm{DM}} h^{2}}{0.12}=5.3 \times 10^{21} \lambda_{h \pi}^{2} \frac{M_{\pi}}{\mathrm{GeV}},
$$

where $\lambda_{h \pi} v_{\mathrm{EW}}$ is the coupling of the Higgs to two dark pions and is given by

$$
\lambda_{h \pi}=\frac{8 \lambda_{1} \lambda_{2} \lambda_{6}}{\lambda_{5} \lambda_{6}^{2}+m^{2} /\left(2 \eta^{2}\right)}
$$

In our model, $\pi \pi \rightarrow \pi \pi$ scattering cross section gets contribution from the contact interaction $\Pi^{4}$ term as well as due to exchange of the three physical scalars $\chi_{1}, \chi_{2}$, and $\chi_{3}$. The interaction of dark pions with $\chi_{1}$ and $\chi_{3}$ is very small and hence we only need to include the contributions due to $s, t$, and $u$ channel exchange of $\chi_{2}$. At low energies, these give contributions to the scattering amplitude which are proportional to that obtained from the self-interaction $\Pi^{4}$. The final result is found to be

$$
\frac{\sigma_{\pi}}{M_{\pi}}=\frac{9 \tilde{\lambda}_{5}^{2}}{32 \pi} \frac{1}{M_{\pi}^{3}},
$$

where $\tilde{\lambda}_{5}=3 \lambda_{5} / 4$.

\section{RESULTS: FREEZE-OUT SCENARIO}

The direct detection of dark matter with nucleons imposes a very strong constraint on the SI scattering cross section of pions, as seen in Fig. 3. This constraint is found to be much stronger in comparison with the indirect detection constraints. The relevant cross sections for this case are shown in Figs. 3(b)-3(f). These have peaks at the Breit-Wigner resonance of $\chi_{1}, \chi_{2}$, and $\chi_{3}$, but exactly at the resonance the pion relic density is significantly small and hence the indirect detection bounds at the resonance 


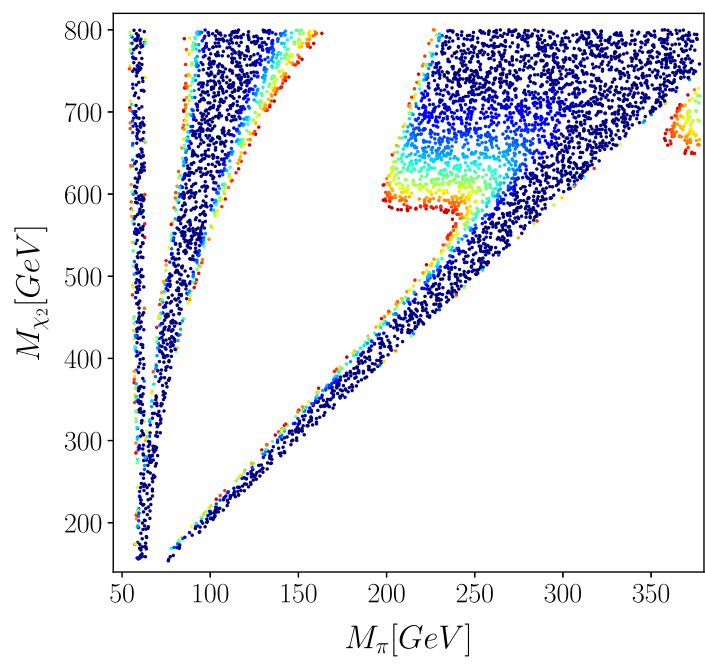

(a)
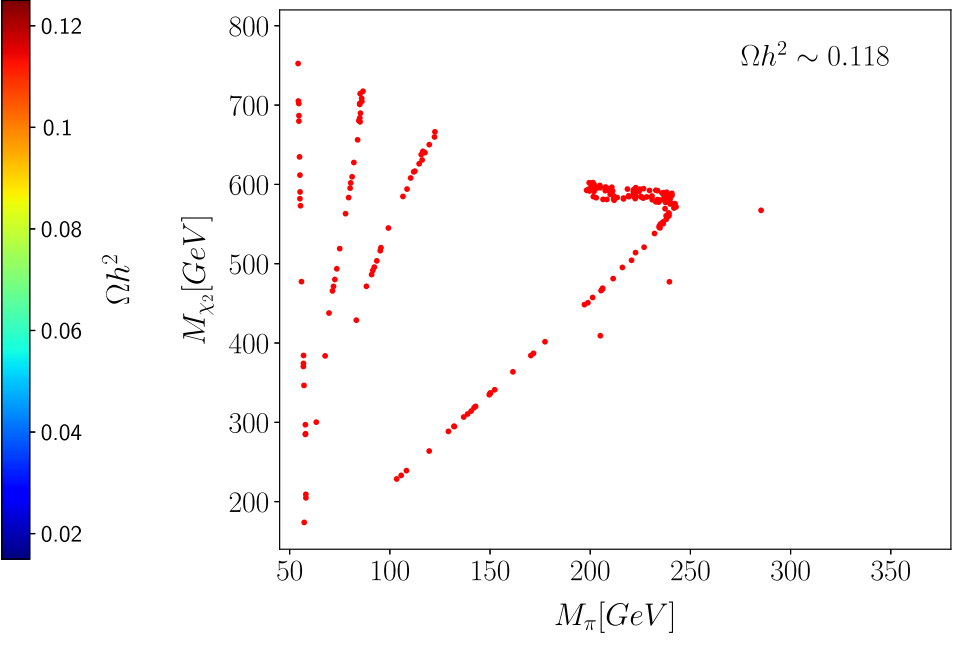

(b)

FIG. 4. Set 1 : in (a), we use the $\Omega h^{2} \leq 0.118$ for $\alpha_{1}=0.01$ radians, $\alpha_{2}=0.1$ radians, and $\alpha_{3}=1.0$ radians showing the resonance effect (color bar shows the relic density). (b) shows the allowed parameter space for $\alpha_{1}=0.01$ radians, $\alpha_{2}=0.1$ radians, and $\alpha_{3}=1.0$ radians which satisfies $\Omega h^{2} \sim 0.118$, direct and indirect detection constraints and collider constraints.

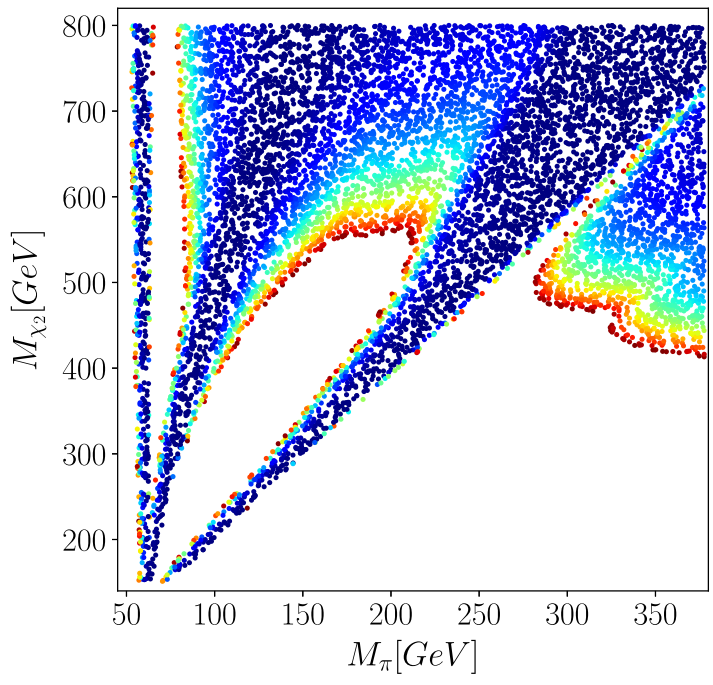

(a)

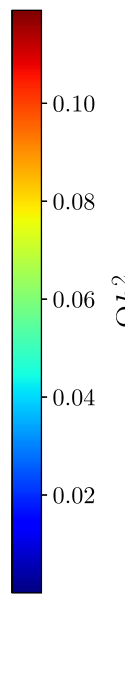

FIG. 5. Set 2: same as Fig. 4 with $\alpha_{1}=0.02$ radians, $\alpha_{2}=0.1$ radians, and $\alpha_{3}=1.0$ radians. become unimportant. ${ }^{2}$ These bounds are also easily satisfied in the off-resonant region where the relic density is $\sim 0.118$ since the cross sections become very small. Hence, we find that, once we apply the direct detection constraints on the DM-nucleon cross sections, the indirect detection

\footnotetext{
${ }^{2}$ We should note that the direct detection and indirect detction constraint can be applied only when the relic density is $\sim 0.118$. For situations where $\Omega h_{\text {model }}^{2}<0.118$, one has to introduce the scale factor $\xi=\Omega h_{\text {model }}^{2} / \Omega h_{\mathrm{CDM}}^{2}$ for direct detection and $\xi^{2}$ for indirect detection [74]. In our case we used the direct and indirect detection constraint only for $\xi$ close to 1 .
}

constraints are automatically satisfied. We also see sudden dips in the cross section in Figs. 3(b)-3(f) for some values of the dark pion mass. These arise since the coupling factors, such as $g_{\chi_{2} \pi \pi}$, cross zero at these values of the dark pion mass.

In Figs. 4(a), 5(a), 6(a), and 7(a), we show the parameter space which satisfies $\Omega h^{2} \leq 0.118$ for the choice of parameters corresponding to Sets 1, 2, 3, and 4, respectively, as given in Table I. The color bar shows the relic density. We find that there are three strips along the resonances arising due to exchange of particles $\chi_{1}, \chi_{2}$, and $\chi_{3}$. In Figs. 5(a) and 7(a), we find that two of these 


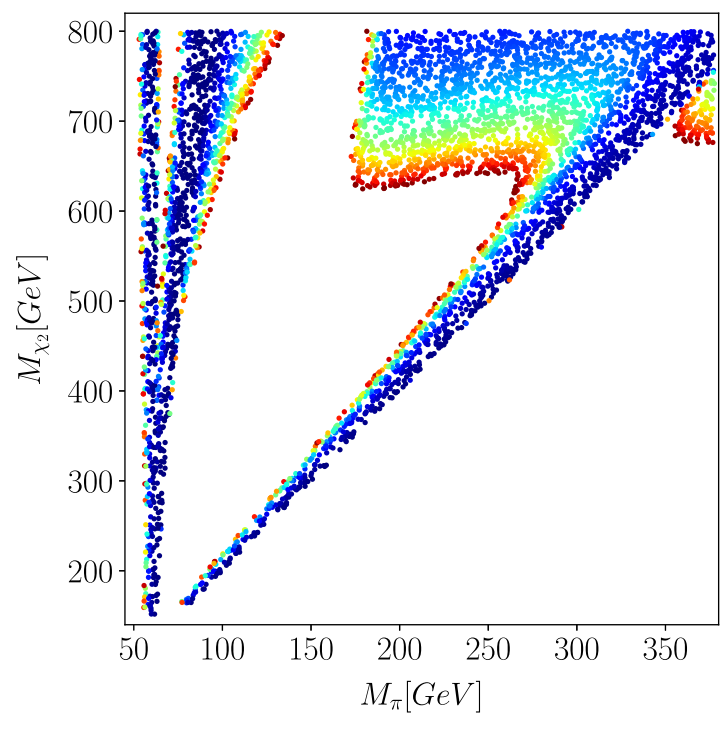

(a)
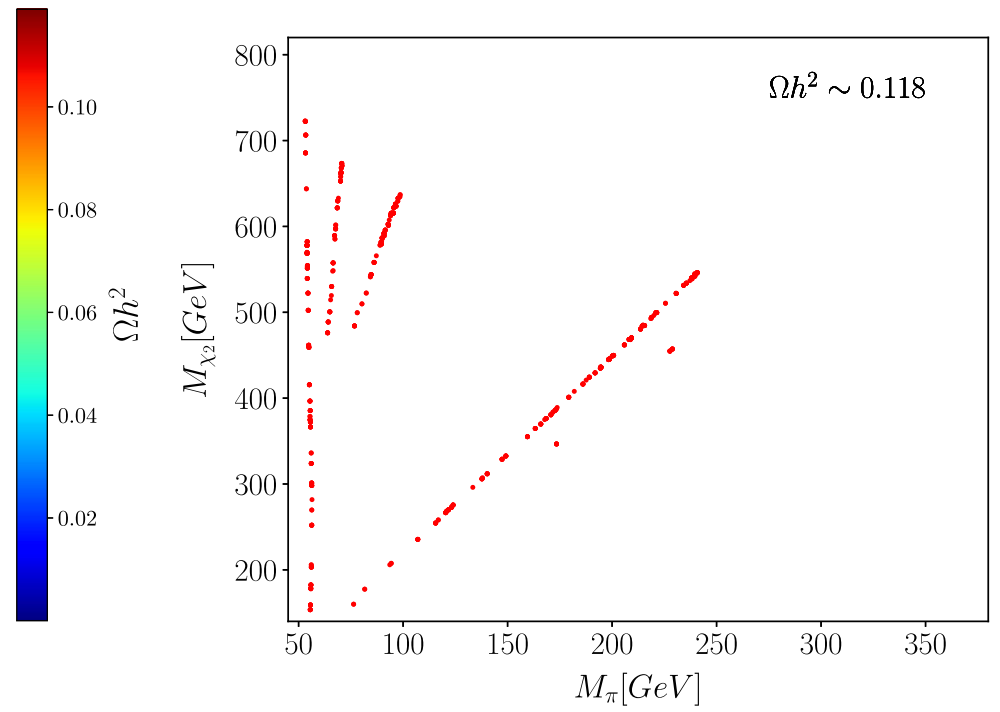

(b)

FIG. 6. Set 3: same as Fig. 4 with $\alpha_{1}=0.01$ radians, $\alpha_{2}=0.2$ radians, and $\alpha_{3}=1.0$ radians.

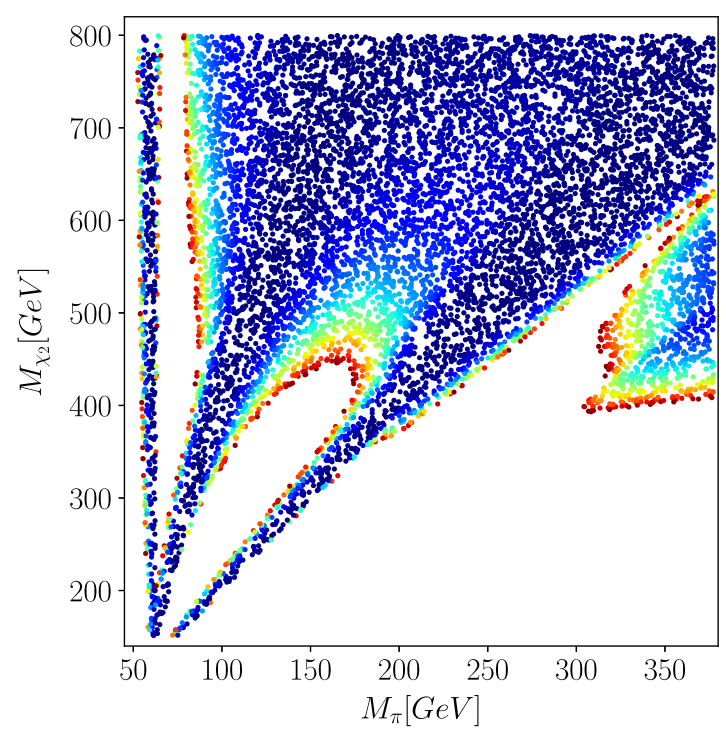

(a)
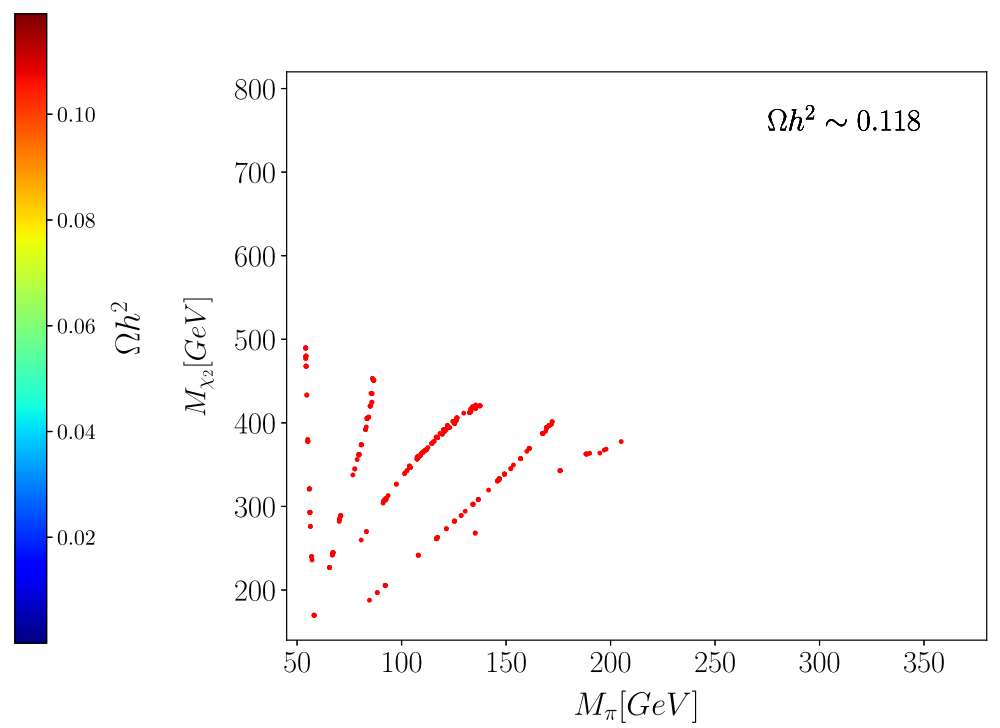

(b)

FIG. 7. Set 4: Same as Fig. 4 with $\alpha_{1}=0.01$ radians, $\alpha_{2}=0.1$ radians, and $\alpha_{3}=0.5$.

TABLE I. Different choices of mixing angles $\alpha_{1}, \alpha_{2}$, and $\alpha_{3}$.

\begin{tabular}{lccc}
\hline \hline & \multicolumn{3}{c}{ Mixing angles in radians } \\
\cline { 2 - 4 } Benchmark points & $\alpha_{1}$ & $\alpha_{2}$ & $\alpha_{3}$ \\
\hline Set 1 & 0.01 & 0.1 & 1.0 \\
Set 2 & 0.02 & 0.1 & 1.0 \\
Set 3 & 0.01 & 0.2 & 1.0 \\
Set 4 & 0.01 & 0.1 & 0.5 \\
\hline \hline
\end{tabular}

strips merge with one another. For these parameter sets (Sets 2 and 4), we also find a much larger number of points which deviate from resonance. The relic density falls sharply when the dark pion is at the resonance of any of the scalars, $\chi_{1}, \chi_{2}$, and $\chi_{3}$ (i.e., $M_{\pi} \sim M_{\chi_{1}, \chi_{2}, \chi_{3}} / 2$ ). The red points are the parameters for which the relic density is close to 0.118. In Figs. 4(b), 5(b), 6(b), and 7(b), we show the allowed parameter space in the $M_{\pi}$ and $M_{\chi_{2}}$ plane for parameter Sets 1, 2, 3, and 4 (Table I), respectively, which satisfies $\Omega h^{2} \sim 0.118$ and direct detection constraint of 
XENON1T. We see that a considerable parameter range gets eliminated due to the direct detection constraints. As mentioned earlier, constraints due to indirect detection are weaker and get automatically satisfied. In the final allowed parameter space [see Figs. 4(b), 5(b), 6(b), and 7(b)], we find that a large number of points lie close to the resonance of the three scalar particles. However, in Fig. 4(b), we also see considerable parameter space for which $M_{\pi}$ deviates considerably from $M_{\chi_{2}} / 2$.

We next scan the entire parameter space corresponding to the three mixing angles and the masses $M_{\pi}$ and $M_{\chi_{2}}$ by randomly selecting parameters over the range $\alpha_{1}, \alpha_{2} \in$ $\left[10^{-4}, 0.35\right]$ radians, $\alpha_{3} \in\left[10^{-2}, 1.55\right]$ radians, $M_{\chi_{2}} \in[150$, 800] $\mathrm{GeV}$, and $M_{\pi} \in[50,400] \mathrm{GeV}$. The mixing angles are taken in this range since they automatically satisfy the collider constraints, as shown in Fig. 1(b). After imposing all the remaining constraints, the final allowed values of $M_{\pi}$ and $M_{\chi_{2}}$ are shown in Fig. 8. We see that the model has sufficiently large allowed parameter space which satisfies all the phenomenological constraints. Some regions in the $M_{\pi}-M_{\chi_{2}}$ plane are found to have higher density of points in comparison to others. However, as we explain below, almost all of this parameter space is allowed. Some regions of this plot arise due to contributions from close to resonant scattering with s-channel exchange of $\chi_{1}, \chi_{2}$, and $\chi_{3}$ in similarity to Figs. 4-7. As expected, we do not get the right relic density exactly at resonance but slightly away from it. For small $M_{\chi_{2}}$, we see a considerably high density of points. These arise due to nonresonant contribution. We point out that the density of points is low near a resonance since in this case the relic density shows a strong dependence on parameters and in order to satisfy all constraints the

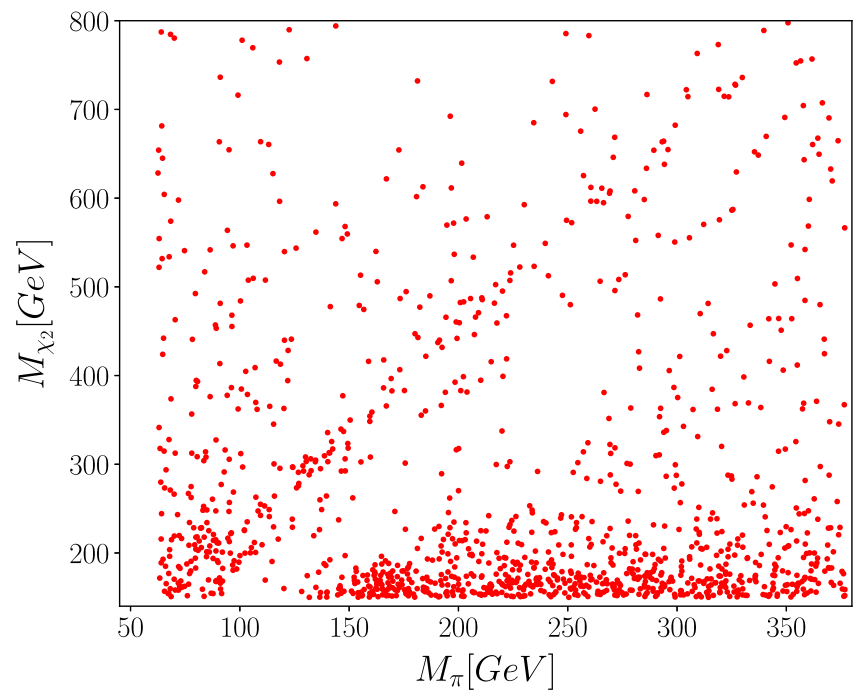

FIG. 8. Allowed parameters in $M_{\pi}-M_{\chi_{2}}$ plane satisfying $\Omega h^{2} \sim 0.118$, direct and indirect detection constraints, and collider constraints. The mixing angles are chosen randomly over the range allowed by the collider constraint, as explained in text. parameters need to be fine-tuned. This does not apply in nonresonant regions where the cross section shows a relatively mild dependence on model parameters. The only forbidden regions are those corresponding to $M_{\pi}<$ $60 \mathrm{GeV}$ and a very narrow strip right along the resonance corresponding to any one of the three scalars. Other than that all regions are allowed. This applies even to some of the gaps seen in Fig. 8 for $M_{\pi}>60 \mathrm{GeV}$. We have explicitly verified this by carefully exploring the parameter space corresponding to these regions.

As mentioned earlier, our phenomenological analysis bears some resemblance to the Higgs portal DM, and a substantial analysis of Higgs portal DM is done before. The simplest yet most popular DM model of scalar singlet extension of SM is studied in depth for $\Omega h^{2} \leq 0.118$ in $[74,75]$ which rules out a vast region $\left(M_{\mathrm{DM}} \leq 500 \mathrm{GeV}\right)$ of parameter space because of strong direct and indirect detection constraints and Higgs invisible decay constraint. As mentioned in [74], results from XENON1T and LZ [76] might exclude a significantly large parameter space of the scalar singlet DM model apart from a very narrow region close to the Higgs resonance $\left(M_{\mathrm{DM}} \sim M_{h_{\mathrm{SM}}} / 2\right)$ [77]. However, our model naturally has a light dark pion and two more Higgs like scalars. Due to the presence of these heavier scalars $\chi_{2}$ and $\chi_{3}$, we find that a significant parameter space opens up both close to and away from the resonance of these scalars.

We next consider the constraint given in Eq. (27). For dark matter particles in the mass range considered in this section implies a very large DM-DM scattering cross section. For the allowed parameter range, we find that the largest value is many orders of magnitude smaller than that given in Eq. (27). This is to be expected in any model based on the freeze-out scenario and is only possible in a freeze-in scenario which allows for much smaller masses.

\section{A. Results: Freeze-in scenario}

The basic formulas for the case of freeze-in scenario are given in Sec. IV. In Fig. 9, we show the relationship between the mass of dark pion and the dark sector particle $\chi_{2}$ for the allowed parameter range which leads to the observed dark matter relic density and is also consistent with the constraint on the dark matter scattering cross section given in Eq. (28) for different values of the parameters $\lambda_{6}$ and $m / \eta$. Here we have set $\lambda=0.2$ and varied the parameter $\lambda_{7}$. Furthermore, we have used Eqs. (32)-(34) and have set the value of $\sigma_{\pi} / M_{\pi}$ equal to its upper limit given by Eq. (28). Hence, the value of $M_{\chi_{2}}$ can only take values below the lines shown in Fig. 9 for the corresponding values of the parameters $\lambda_{6}$ and $m / \eta$. Larger values of this mass are ruled out by astrophysical observations. We point out that $M_{\chi_{2}}$ is directly proportional to the self-coupling $\lambda_{5}$ and hence the region above the lines shown in Fig. 9 correspond to values of $\lambda_{5}$ ruled out by observations. 


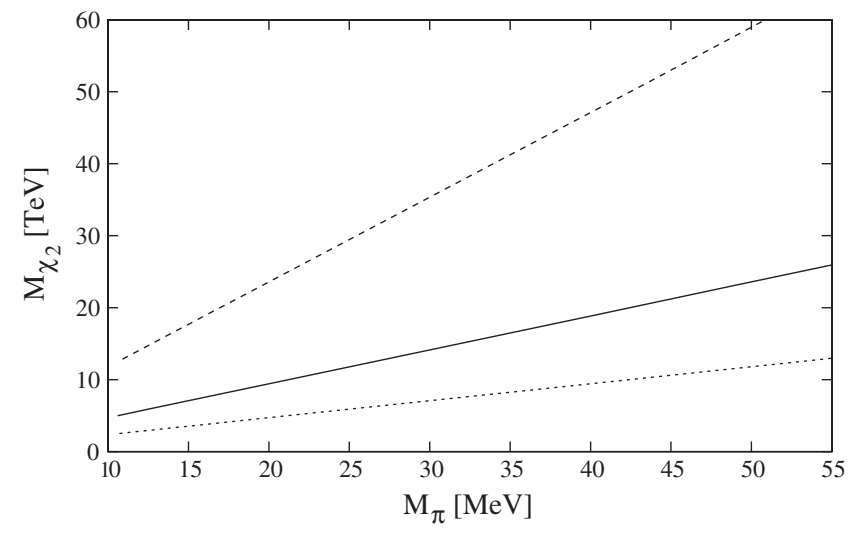

FIG. 9. The relationship between the dark pion mass $M_{\pi}$ and the $\chi_{2}$ meson mass $M_{\chi_{2}}$ for the freeze-in scenario. The short dashed, solid, and long dashed lines correspond to parameter values $\left(\lambda_{6}=0.5 \times 10^{-4}, m / \eta=0.5\right),\left(\lambda_{6}=10^{-4}, m / \eta=0.5\right)$, and $\left(\lambda_{6}=10^{-4}, m / \eta=0.2\right)$, respectively. We have terminated the parameter range at $M_{\pi}=55 \mathrm{MeV}$. For larger values of this mass, the strong sector coupling $\lambda_{5}$ becomes very large and our calculation is not reliable.

In the limit $\lambda_{5} \lambda_{6}^{2} \ll m^{2} /\left(2 \eta^{2}\right)$, we find that the relationship between $M_{\pi}$ and $M_{\chi_{2}}$ is approximately linear for fixed values of $\lambda_{6}$ and $m / \eta$, as seen in Fig. 9. In this figure, we have restricted the dark pion mass $M_{\pi}$ to be less than $55 \mathrm{MeV}$. For larger values, the strong sector coupling $\lambda_{5}$ becomes very large and our leading order calculation of $\pi \pi$ scattering becomes unreliable. For a dark pion mass of $30 \mathrm{MeV}$, we find that $\lambda_{5} \approx 1.5$, which may be considered perturbative. We find that the experimental constraint on the Higgs decay to visible sector particles is easily satisfied for all the values shown in Fig. 9. For this entire range of parameters, the mass of dark sector particle $\chi_{3}$ is considerably larger than that of $\chi_{2}$.

\section{CONCLUSION}

We have studied the DM constraints within a conformal extension of the Standard Model of particle physics. The model has strongly coupled dark sector which triggers the electroweak symmetry breaking. The model leads to three neutral scalars $\chi_{1}, \chi_{2}$, and $\chi_{3}$ and we identify $\chi_{1}$ as the observed Higgs boson with mass $125 \mathrm{GeV}$. The model also predicts a pseudoscalar particle which we assume is the DM candidate and refer to it as a dark pion. We perform all our calculations using an effective Lagrangian for the strongly coupled dark sector. We first assume the freeze-out scenario for obtaining the dark matter relic density. We find that the observed relic density $\Omega h^{2}=0.1187 \pm 0.0017$ is obtained for a considerable range of parameter space, some of which lies close to the resonance of any one of the neutral scalars. We find that the model is also able to explain the constraints due to direct and indirect detection of DM and the collider constraints. The collider constraints impose limits on the mixing angles of the neutral scalars. The upper bound on the SI DM-nucleon cross section obtained from direct detection experiments, such as XENON1T, imposes a strong constraint on the model. The bounds arising due to searches of gamma ray signal from the galactic center and dSphs are found to be not very stringent and do not impose any additional constraint on the parameter space. The dark pions have significant self-interaction. However, within the freeze-out scenario, the value of $\sigma_{\mathrm{DM}} / M_{\mathrm{DM}}$ turns out to be relatively small in comparison to the preferred astrophysical value. This is due to the large value of mass $M_{\mathrm{DM}}$ required in this framework.

We have also studied the implications of the conformal model assuming a freeze-in scenario for relic dark matter density. We argue that the model naturally leads to a very low mass dark pion while maintaining a relatively large mass scale of dark sector strong interactions. This is due to the fact that the dark pion mass is controlled by chiral symmetry breaking terms in the dark sector. We can choose this terms very small without fine-tuning. We find that within the freeze-in scenario the model is able to explain all astrophysical and collider observations. As expected in this case, we can also choose $\sigma_{\pi} / M_{\pi}$ close to the value preferred by astrophysical observations.

In our analysis, we have assumed only a single species of dark fermions which lead to only a single candidate dark pion. Additional dark fermions and hence dark pions can also be added in our model. This may open up additional regions in the parameter space.

\section{ACKNOWLEDGMENTS}

P. S. and P. J. acknowledge funding from the Science and Engineering Research Board (SERB), Government of India.
[1] P. A. R. Ade et al., Planck 2015 results. XIII. Cosmological parameters, Astron. Astrophys. 594, A13 (2016).

[2] D. S. Akerib et al., Results from a Search for Dark Matter in the Complete LUX Exposure, Phys. Rev. Lett. 118, 021303 (2017).
[3] D.S. Akerib et al., Improved Limits on Scattering of Weakly Interacting Massive Particles from Reanalysis of 2013 LUX Data, Phys. Rev. Lett. 116, 161301 (2016).

[4] X. Cui et al., Dark Matter Results from 54-Ton-Day Exposure of PandaX-II Experiment, Phys. Rev. Lett. 119, 181302 (2017). 
[5] A. Tan et al., Dark Matter Results from First 98.7 Days of Data from the PandaX-II Experiment, Phys. Rev. Lett. 117, 121303 (2016).

[6] E. Aprile et al., Dark Matter Search Results from a One TonYear Exposure of XENON1T, Phys. Rev. Lett. 121, 111302 (2018).

[7] R. Agnese et al., New Results from the Search for LowMass Weakly Interacting Massive Particles with the CDMS Low Ionization Threshold Experiment, Phys. Rev. Lett. 116, 071301 (2016).

[8] G. Angloher et al., Results on light dark matter particles with a low-threshold CRESST-II detector, Eur. Phys. J. C 76, 25 (2016).

[9] T. Daylan, D. P. Finkbeiner, D. Hooper, T. Linden, S. K. N. Portillo, N. L. Rodd, and T. R. Slatyer, The characterization of the gamma-ray signal from the central Milky Way: A case for annihilating dark matter, Phys. Dark Universe 12, 1 (2016).

[10] M. Ackermann et al., The Fermi galactic center GeV excess and implications for dark matter, Astrophys. J. 840, 43 (2017).

[11] M. Ackermann et al., Updated search for spectral lines from Galactic dark matter interactions with pass 8 data from the Fermi large area telescope, Phys. Rev. D 91, 122002 (2015).

[12] M. L. Ahnen et al. (MAGIC Collaboration), Limits to dark matter annihilation cross-section from a combined analysis of MAGIC and Fermi-LAT observations of dwarf satellite galaxies, J. Cosmol. Astropart. Phys. 02 (2016) 039.

[13] K. A. Meissner and H. Nicolai, Conformal symmetry and the Standard Model, Phys. Lett. B 648, 312 (2007).

[14] K. A. Meissner and H. Nicolai, Effective action, conformal anomaly and the issue of quadratic divergences, Phys. Lett. B 660, 260 (2008).

[15] T. Hur and P. Ko, Scale Invariant Extension of the Standard Model with Strongly Interacting Hidden Sector, Phys. Rev. Lett. 106, 141802 (2011).

[16] G. Kashyap, S. Mitra, and P. Jain, Non-relativistic matter and dark energy in a quantum conformal model, Astropart. Phys. 75, 64 (2016).

[17] P. Jain, G. Kashyap, and S. Mitra, The fine tuning of the cosmological constant in a conformal model, Int. J. Mod. Phys. A 30, 1550171 (2015).

[18] D. N. Spergel and P. J. Steinhardt, Observational Evidence for Self-interacting Cold Dark Matter, Phys. Rev. Lett. 84, 3760 (2000).

[19] M. C. Bento, O. Bertolami, and R. Rosenfeld, Cosmological constraints on an invisibly decaying Higgs boson, Phys. Lett. B 518, 276 (2001).

[20] R. Massey et al., The behaviour of dark matter associated with four bright cluster galaxies in the $10 \mathrm{kpc}$ core of Abell 3827, Mon. Not. R. Astron. Soc. 449, 3393 (2015).

[21] D. Harvey, R. Massey, T. Kitching, A. Taylor, and E. Tittley, The non-gravitational interactions of dark matter in colliding galaxy clusters, Science 347, 1462 (2015).

[22] F. Kahlhoefer, K. Schmidt-Hoberg, J. Kummer, and S. Sarkar, On the interpretation of dark matter self-interactions in Abell 3827, Mon. Not. R. Astron. Soc. 452, L54 (2015).

[23] Y. Hochberg, E. Kuflik, H. Murayama, T. Volansky, and J. G. Wacker, Model for Thermal Relic Dark Matter of
Strongly Interacting Massive Particles, Phys. Rev. Lett. 115, 021301 (2015).

[24] M. Kaplinghat, S. Tulin, and H.-B. Yu, Dark Matter Halos as Particle Colliders: Unified Solution to Small-Scale Structure Puzzles from Dwarfs to Clusters, Phys. Rev. Lett. 116, 041302 (2016).

[25] M. Heikinheimo and C. Spethmann, Galactic centre GeV photons from dark technicolor, J. High Energy Phys. 12 (2014) 084.

[26] J. C. Collins, A. Duncan, and S. D. Joglekar, Trace and dilatation anomalies in gauge theories, Phys. Rev. D 16, 438 (1977).

[27] R. Gomm, P. Jain, R. Johnson, and J. Schechter, Scale anomaly and the scalars, Phys. Rev. D 33, 801 (1986).

[28] A. Alloul, N. D. Christensen, C. Degrande, C. Duhr, and B. Fuks, FeynRules 2.0-A complete toolbox for tree-level phenomenology, Comput. Phys. Commun. 185, 2250 (2014).

[29] J. McDonald, Gauge singlet scalars as cold dark matter, Phys. Rev. D 50, 3637 (1994).

[30] C. P. Burgess, M. Pospelov, and T. ter Veldhuis, The minimal model of nonbaryonic dark matter: A singlet scalar, Nucl. Phys. B619, 709 (2001).

[31] G. Arcadi, A. Djouadi, and M. Raidal, Dark matter through the Higgs portal, arXiv:1903.03616.

[32] P. Athron et al., Global analyses of Higgs portal singlet dark matter models using GAMBIT, Eur. Phys. J. C 79, 38 (2019).

[33] P. Athron et al., Status of the scalar singlet dark matter model, Eur. Phys. J. C 77, 568 (2017).

[34] T. A. Collaboration, Combined measurements of Higgs boson production and decay using up to $80 \mathrm{fb}^{-1}$ of protonproton collision data at $\sqrt{s}=13 \mathrm{TeV}$ collected with the ATLAS experiment, Technical Report No. ATLAS-CONF2018-031, CERN, Geneva, 2018.

[35] A. M. Sirunyan et al., Combined measurements of Higgs boson couplings in proton-proton collisions at $\sqrt{\mathrm{s}}=$ 13 TeV, Eur. Phys. J. C 79, 421 (2019).

[36] P. Bechtle, S. Heinemeyer, O. Stål, T. Stefaniak, and G. Weiglein, HiggsSignals: Confronting arbitrary Higgs sectors with measurements at the Tevatron and the LHC, Eur. Phys. J. C 74, 2711 (2014).

[37] V. Khachatryan et al., Searches for invisible decays of the Higgs boson in pp collisions at $\sqrt{s}=7,8$, and $13 \mathrm{TeV}$, J. High Energy Phys. 02 (2017) 135.

[38] M. Aaboud et al., Combination of Searches for Invisible Higgs Boson Decays with the ATLAS Experiment, Phys. Rev. Lett. 122, 231801 (2019).

[39] A. Djouadi, J. Kalinowski, and M. Spira, HDECAY: A program for Higgs boson decays in the standard model and its supersymmetric extension, Comput. Phys. Commun. 108, 56 (1998).

[40] A. Belyaev, N. D. Christensen, and A. Pukhov, CalcHEP 3.4 for collider physics within and beyond the Standard Model, Comput. Phys. Commun. 184, 1729 (2013).

[41] P. Gondolo and G. Gelmini, Cosmic abundances of stable particles: Improved analysis, Nucl. Phys. B360, 145 (1991).

[42] J. Edsjo and P. Gondolo, Neutralino relic density including coannihilations, Phys. Rev. D 56, 1879 (1997).

[43] G. Bélanger, F. Boudjema, A. Pukhov, and A. Semenov, micrOMEGAs4.1: Two dark matter candidates, Comput. Phys. Commun. 192, 322 (2015). 
[44] K. Griest and D. Seckel, Three exceptions in the calculation of relic abundances, Phys. Rev. D 43, 3191 (1991).

[45] M. Ibe, H. Murayama, and T. T. Yanagida, Breit-Wigner enhancement of dark matter annihilation, Phys. Rev. D 79, 095009 (2009).

[46] W.-L. Guo and Y.-L. Wu, Enhancement of dark matter annihilation via Breit-Wigner resonance, Phys. Rev. D 79, 055012 (2009).

[47] M. Duch and B. Grzadkowski, Resonance enhancement of dark matter interactions: The case for early kinetic decoupling and velocity dependent resonance width, J. High Energy Phys. 09 (2017) 159.

[48] J. M. Cline, K. Kainulainen, P. Scott, and C. Weniger, Update on scalar singlet dark matter, Phys. Rev. D 88, 055025 (2013); Erratum, Phys. Rev. D 92, 039906(E) (2015).

[49] L. Feng, S. Profumo, and L. Ubaldi, Closing in on singlet scalar dark matter: LUX, invisible Higgs decays and gamma-ray lines, J. High Energy Phys. 03 (2015) 045.

[50] J. F. Gunion, H. E. Haber, G. L. Kane, and S. Dawson, The Higgs Hunter's guide, Front. Phys. 80, 1 (2000).

[51] A. Djouadi, The anatomy of electro-weak symmetry breaking. I: The Higgs boson in the standard model, Phys. Rep. 457, 1 (2008).

[52] S. Dittmaier et al., Handbook of LHC Higgs cross sections: 1. Inclusive observables, arXiv:1101.0593.

[53] J. F. Navarro, C. S. Frenk, and S. D. M. White, The Structure of cold dark matter halos, Astrophys. J. 462, 563 (1996).

[54] J. Einasto, On constructing models of stellar systems. V. The binomial model, Publ. Tartu Astrofizica Observatory 36, 414 (1968).

[55] R. A. Flores and J. R. Primack, Observational and theoretical constraints on singular dark matter halos, Astrophys. J. 427, L1 (1994).

[56] A. H. G. Peter, M. Rocha, J. S. Bullock, and M. Kaplinghat, Cosmological simulations with self-interacting dark matter II: Halo shapes vs observations, Mon. Not. R. Astron. Soc. 430, 105 (2013).

[57] M. Rocha, A. H. G. Peter, J. S. Bullock, M. Kaplinghat, S. Garrison-Kimmel, J. Onorbe, and L. A. Moustakas, Cosmological simulations with self-interacting dark matter I: Constant density cores and substructure, Mon. Not. R. Astron. Soc. 430, 81 (2013).

[58] O. D. Elbert, J. S. Bullock, S. Garrison-Kimmel, M. Rocha, J. Oñorbe, and A.H. G. Peter, Core formation in dwarf haloes with self-interacting dark matter: No fine-tuning necessary, Mon. Not. R. Astron. Soc. 453, 29 (2015).

[59] R. Dave, D. N. Spergel, P. J. Steinhardt, and B. D. Wandelt, Halo properties in cosmological simulations of selfinteracting cold dark matter, Astrophys. J. 547, 574 (2001).

[60] S. Garrison-Kimmel, M. Boylan-Kolchin, J. S. Bullock, and E. N. Kirby, Too big to fail in the local group, Mon. Not. R. Astron. Soc. 444, 222 (2014).

[61] M. Boylan-Kolchin, J. S. Bullock, and M. Kaplinghat, Too big to fail? The puzzling darkness of massive Milky Way subhaloes, Mon. Not. R. Astron. Soc. Lett. 415, L40 (2011).
[62] J. F. Navarro, C. S. Frenk, and S. D. M. White, A universal density profile from hierarchical clustering, Astrophys. J. 490, 493 (1997).

[63] S.-H. Oh, C. Brook, F. Governato, E. Brinks, L. Mayer, W. J. G. de Blok, A. Brooks, and F. Walter, The central alope of dark matter cores in Dwarf Galaxies: Simulations versus things, Astron. J. 142, 24 (2011).

[64] M. G. Walker and J. Peñarrubia, A method for measuring (slopes of) the mass profiles of dwarf spheroidal galaxies, Astrophys. J. 742, 20 (2011).

[65] S. W. Randall, M. Markevitch, D. Clowe, A. H. Gonzalez, and M. Bradac, Constraints on the self-interaction crosssection of dark matter from numerical simulations of the merging Galaxy cluster 1E 0657-56, Astrophys. J. 679, 1173 (2008).

[66] M. Markevitch, A. H. Gonzalez, D. Clowe, A. Vikhlinin, L. David, W. Forman, C. Jones, S. Murray, and W. Tucker, Direct constraints on the dark matter self-interaction crosssection from the merging galaxy cluster 1E0657-56, Astrophys. J. 606, 819 (2004).

[67] R. Campbell, S. Godfrey, H. E. Logan, A. D. Peterson, and A. Poulin, Implications of the observation of dark matter self-interactions for singlet scalar dark matter, Phys. Rev. D 92, 055031 (2015).

[68] L. J. Hall, K. Jedamzik, J. March-Russell, and S. M. West, Freeze-in production of FIMP dark matter, J. High Energy Phys. 03 (2010) 080.

[69] K. S. Babu and R. N. Mohapatra, 7 keV scalar dark matter and the anomalous galactic X-ray spectrum, Phys. Rev. D 89, 115011 (2014).

[70] G. Bélanger, F. Boudjema, A. Goudelis, A. Pukhov, and B. Zaldivar, micrOMEGAs5.0: Freeze-in, Comput. Phys. Commun. 231, 173 (2018).

[71] N. Bernal, M. Heikinheimo, T. Tenkanen, K. Tuominen, and V. Vaskonen, The dawn of FIMP dark matter: A review of models and constraints, Int. J. Mod. Phys. A 32, 1730023 (2017).

[72] Z. Kang, View FImP miracle (by scale invariance) à la selfinteraction, Phys. Lett. B 751, 201 (2015).

[73] N. Bernal, C. Cosme, and T. Tenkanen, Phenomenology of self-interacting dark matter in a matter-dominated Universe, Eur. Phys. J. C 79, 99 (2019).

[74] J. A. Casas, D. G. Cerdeño, J. M. Moreno, and J. Quilis, Reopening the Higgs portal for single scalar dark matter, J. High Energy Phys. 05 (2017) 036.

[75] M. Escudero, A. Berlin, D. Hooper, and M.-X. Lin, Toward (Finally!) ruling out $\mathrm{Z}$ and Higgs mediated dark matter models, J. Cosmol. Astropart. Phys. 12 (2016) 029.

[76] D. S. Akerib et al., Projected WIMP sensitivity of the LUXZEPLIN (LZ) dark matter Experiment, arXiv:1802.06039.

[77] P. Athron, J. M. Cornell, F. Kahlhoefer, J. Mckay, P. Scott, and S. Wild, Impact of vacuum stability, perturbativity and XENON1T on global fits of $\mathbb{Z}_{2}$ and $\mathbb{Z}_{3}$ scalar singlet dark matter, Eur. Phys. J. C 78, 830 (2018). 